\title{
EDT dal numero 1/2017 sarà Open Access
}

Caro lettore, cara lettrice,

La Rivista Economia e Diritto del Terziario del Gruppo Banca Carige con il prossimo anno si rinnova.

Dal primo fascicolo del 2017, per accrescere la sua diffusione in ambito scientifico, economico, sociale e culturale, promuovere la sua progressiva internazionalizzazione e corrispondere alle esigenze conoscitive di studiosi, esperti, imprenditori e dirigenti, Economia e diritto del terziario passerà alla pubblicazione in Open Access che consente l'accesso libero e aperto ai contenuti.

Per poter scaricare senza oneri di acquisto gli articoli di ogni fascicolo sarà quindi sufficiente collegarsi o al sito di Banca Carige (http://www.gruppocarige.it)

o al sito di FrancoAngeli (http://www.francoangeli.it) alla pagina della Rivista.

Buona lettura

Il Direttore

Mario Venturino
Il Direttore Responsabile

Prof. Amedeo Amato 